\title{
SUGAWARA CONSTRUCTION FOR HIGHER GENUS RIEMANN SURFACES ${ }^{1}$
}

\author{
Martin Schlichenmaier \\ Department of Mathematics and Computer Science \\ University of Mannheim \\ D-68131 Mannheim, Germany \\ E-mail:schlichenmaier@math.uni-mannheim.de
}

\begin{abstract}
By the classical genus zero Sugawara construction one obtains from admissible representations of affine Lie algebras (Kac-Moody algebras of affine type) representations of the Virasoro algebra. In this lecture first the classical construction is recalled. Then, after giving a review on the global multi-point algebras of Krichever-Novikov type for compact Riemann surfaces of arbitrary genus, the higher genus Sugawara construction is introduced. Finally, the lecture reports on results obtained in joint work with O.K. Sheinman. We were able to show that also in the higher genus, multi-point situation one obtains from representations of the global algebras of affine type representations of a centrally extended algebra of meromorphic vector fields on Riemann surfaces. The latter algebra is the generalization of the Virasoro algebra to higher genus.

Invited lecture at the XVI ${ }^{\text {th }}$ workshop on geometric methods in physics, Bialowieza, Poland, June 30 - July 6, 1997
\end{abstract}

\section{INTRODUCTION}

Two-dimensional conformal field theory has played an important role in theoretical physics for about 20 years now. One of its origins lies in the realm of statistical mechanics, where it is reflected by the scaling invariance of phase transitions. Another of its origins lies in string theory and two-dimensional quantum field theory. Let me mention in this latter context the pioneering work of A.A. Belavin, A. M. Polyakov and A.B. Zamolodchikov [1].

\footnotetext{
${ }^{1}$ Research partially supported by the Volkswagen-Stiftung (RiP-program at Oberwolfach) and a DFG-RFBR cooperation (436 RUS 113/276)
} 
The Sugawara construction [23] is one of the basic constructions in two-dimensional conformal field theory. For example, starting from Wess-Zumino-Novikov-Witten (WZNW) models associated to a simple Lie group $G$ the modes of the energymomentum tensor define a representation of the Virasoro algebra, the centrally extended Lie algebra of vector fields on the circle $S^{1}$.

This has its mathematical interpretation in the representation theory of affine Lie algebras (Kac-Moody algebras of untwisted affine type). If one interprets the affine Lie algebra as Lie algebra of the loop group $L G$, i.e. of the group of maps from $S^{1}$ to the group $G$, and the algebra of vector fields on $S^{1}$ as Lie algebra of the group $\operatorname{Diff}^{+}\left(S^{1}\right)$ of orientation preserving diffeomorphisms on $S^{1}$ one obtains as an integrated version of the Sugawara construction the fact that every positive energy representation of the loop group admits a projective intertwiner action of $\operatorname{Diff}^{+}\left(S^{1}\right)$. For more information, general background, and references to the original (mathematical) literature let me refer to the book of A. Pressley and G. Segal [14, $\S 13]$. For references to the works of physicists see the review [9].

The Sugawara construction above is the classical genus zero construction in the sense that these vector fields on $S^{1}$ which are finite linear combinations of Fourier modes can be extended to meromorphic vector fields on the projective line $\mathbb{P}^{1}(\mathbb{C})$, resp. on the sphere $S^{2}$, resp. on the Riemann surface of genus zero, which are holomorphic outside $\{0, \infty\}$.

In the approach to conformal field theory on general compact Riemann surfaces (or equivalently on smooth projective curves over $\mathbb{C}$ ) due to A. Tsuchiya, K. Ueno and Y. Yamada [24] the Sugawara construction is employed locally at every "insertion point" to define the Knizhnik-Zamolodchikov connection for the bundle of conformal blocks over the moduli space of curves. With the help of this connection the authors proved factorization rules and the Verlinde formula by studying the behaviour at the boundary components of the moduli space representing stable, but non-smooth curves. See also the approach of physicists, e.g. T. Eguchi, H. Ooguri [5] and D. Friedan, S. Shenker [6]. For the connection of the Sugawara construction to geometric quantization see for example J.-L. Brylinski, D. McLaughlin [3].

After the above indications about the importance of the Sugawara construction, let me now outline the plan of the lecture. Because the plenary lectures were supposed to be pedagogical in nature and because the main part of the audience might not be very familiar with conformal field theory I will first recall in Section 2 the definition of the Virasoro algebra, the description how one obtains central extensions of Lie algebras, and how the Sugawara construction works in the classical situation, first for the special case of the Heisenberg algebra (the oscillator algebra) and then in the case of general affine Lie algebras. Only these objects from conformal field theory we will need here.

The results presented are completely well-known and classical nowadays. Hence, I will omit nearly all references to the original literature and instead give as reference for further reading and more details the books of V. Kac [11] and V. Kac, A.K. 
Raina [12].

Starting from the genus zero case in the above interpretation, it is quite natural to try to extend the situation to higher genus Riemann surfaces using globally defined meromorphic objects. The global objects, algebras, etc. were introduced by Krichever and Novikov [13] in 1987 for the two-point situation and generalized by me to the multi-point situation [16], [17]. This (non-trivial) generalization is crucial for applications in the theory of conformal blocks. In Section 3 I recall the definition of these objects and the relevant results.

Finally, Section 4 reports on the extension of the Sugawara construction to higher genus Riemann surfaces with the help of the Krichever-Novikov objects. This is joint work with Oleg K. Sheinman from Moscow. We obtain from representations of the global multi-point algebras of affine type representations of a centrally extended algebra of meromorphic vector fields on Riemann surfaces. The latter algebra is the generalization of the Virasoro algebra to higher genus. Details can be found in [20], [19]. See also this section for reference to related work, e.g. [2]. At the moment we are employing the higher genus Sugawara construction on a global operator approach to the theory of conformal blocks [21].

\section{THE CLASSICAL GENUS ZERO SITUATION}

In this section I will recall the definition of the Virasoro algebra and how the classical (genus zero) Sugawara construction works.

\subsection{The Witt Algebra}

Let $S^{1}$ be the unit circle. Denote by $\operatorname{Diff}^{+}\left(S^{1}\right)$ the group of orientation preserving diffeomorphisms $S^{1} \rightarrow S^{1}$. The space of $C^{\infty}$-vector fields $\operatorname{Vect}\left(S^{1}\right)$ can be considered as its Lie algebra. The exponential exp : $\operatorname{Vect}\left(S^{1}\right) \rightarrow \operatorname{Diff}^{+}\left(S^{1}\right)$ is defined by assigning to a vector field the flow it generates. Let me point out that the exponential map is neither locally 1:1 nor locally surjective [14, p.28]. This is quite in contrast to the finite-dimensional situation. Inside the Lie algebra $\operatorname{Vect}\left(S^{1}\right)$ we can consider certain important subalgebras, e.g. the subalgebra of analytic vector fields, or the subalgebra of the vector fields which are sums of finitely many Fourier modes. With the latter subalgebra we will deal in the following. It is convenient to pass to complex-valued vector fields. Let $\varphi$ be the coordinate on $S^{1}$ then the vector fields

$$
l_{m}:=(-\mathrm{i}) \mathrm{e}^{\mathrm{i} m \varphi} \frac{d}{d \varphi}, \quad m \in \mathbb{Z}
$$

correspond to the Fourier modes. One calculates

$$
\left[l_{n}, l_{m}\right]=(m-n) l_{n+m}
$$


The Lie algebra $\mathcal{W}$ generated by (finite) linear combinations of the $l_{m}$ is called the Witt algebra, or the Virasoro algebra without central term. In the complex coordinate $z=\exp (\mathrm{i} \varphi)$ the elements (2.1) can be considered as the restrictions of the meromorphic vector fields

$$
l_{m}:=z^{m+1} \frac{d}{d z}, \quad m \in \mathbb{Z},
$$

which are holomorphic in $\mathbb{C} \backslash\{0\}$. If we take $w=1 / z$ the local coordinate at $\infty \in \mathbb{P}^{1}(\mathbb{C})$ we see with $\frac{d}{d z}=-w^{2} \frac{d}{d w}$ that $l_{m}$ can be represented there as

$$
l_{m}=-w^{1-m} \frac{d}{d w}, \quad m \in \mathbb{Z} .
$$

We can describe $\mathcal{W}$ as the Lie algebra of meromorphic vector fields on $\mathbb{P}^{1}(\mathbb{C})$ which have only poles at $\{0, \infty\}$. This description will be our point of generalization to higher genus.

Note that from (2.3) and (2.4) it follows that the subalgebra of global holomorphic vector fields is the subalgebra

$$
\left\langle l_{-1}, l_{0}, l_{1}\right\rangle_{\mathbb{C}} \cong \operatorname{sl}(2, \mathbb{C})
$$

\subsection{Central Extensions and the Virasoro Algebra}

In the quantization process one is naturally led from representations to projective representations. In our context, let $\Phi: \mathcal{W} \rightarrow \operatorname{End}(V)$ be a realization of the vector fields as linear operators on a vector space $V$, e.g. the quantum Hilbert space of the theory. Typically we obtain in important applications for the commutator in $\operatorname{End}(V)$

$$
\left[\Phi\left(l_{n}\right), \Phi\left(l_{m}\right)\right]=\Phi\left(\left[l_{n}, l_{m}\right]\right)+\gamma\left(l_{n}, l_{m}\right) \cdot i d_{V},
$$

with some bilinear form $\gamma: \mathcal{W} \times \mathcal{W} \rightarrow \mathbb{C}$. If $\gamma=0$ then the realization will be a linear representation of the Lie algebra $\mathcal{W}$. If not, it still will be very often a projective representation. This says that $\gamma$ fulfils additional cocycle conditions, see (2.7). Such projective representations can be described more conveniently as linear representations of a central extension of $\mathcal{W}$. This algebra will be the Virasoro algebra.

In view of the next section let me recall the definition of a central extension of a Lie algebra. Let $L$ be an arbitrary Lie algebra. A bilinear map $\alpha: L \times L \rightarrow \mathbb{C}$ is called a (two-) cocycle if

$$
\begin{gathered}
\alpha(a, b)=-\alpha(b, a), \\
\alpha([a, b], c)+\alpha([b, c], a)+\alpha([c, a], b)=0,
\end{gathered}
$$

for all $a, b, c \in L$. Given such a cocycle a (one-dimensional) central extension $\widehat{L}_{\alpha}$ can be defined by taking as underlying vector space

$$
\widehat{L}_{\alpha}:=\mathbb{C} \oplus L,
$$


and as Lie product

$$
[(r, a),(s, b)]=(\alpha(a, b),[a, b]), \quad r, s \in \mathbb{C}, \quad a, b \in L
$$

By the cocycle condition (2.7) $\widehat{L}_{\alpha}$ is a Lie algebra. If we use the notation $t:=(1,0)$ and $\widehat{a}:=(0, a)$ we can rewrite $(2.9)$ as

$$
[\widehat{a}, \widehat{b}]=\widehat{[a, b}]+\alpha(a, b) \cdot t, \quad a, b \in L, \quad\left[t, \widehat{L}_{\alpha}\right]=0
$$

In particular, $\mathbb{C} \cdot t$ is central in $\widehat{L}_{\alpha}$.

If we compare (2.6) with (2.10) we see that by setting $\Phi(\widehat{a}):=\Phi(a)$ and $\Phi(t):=$ $i d_{V}$ we obtain a linear representation of the centrally extended algebra $\widehat{L}_{\gamma}$ (i.e. for which the cocycle $\gamma$ is used to define the extension).

Central extensions are classified up to equivalence by the elements of the Lie algebra cohomology group $\mathrm{H}^{2}(L, \mathbb{C})$, i.e. by the two-cocycles modulo coboundary. (See [10] for the definition and more background information.) Note, that by multiplying the cocycles with $r \in \mathbb{C}^{*}$ we obtain non-equivalent but isomorphic central extensions. For the Witt algebra one calculates $\mathrm{H}^{2}(\mathcal{W}, \mathbb{C}) \cong \mathbb{C}$ (e.g. see [7], [12]). Hence, there are only two essentially different central extensions. The first one is the trivial, or splitting one, which is given by $\alpha=0$. The second one is obtained for any $\alpha \neq 0$. It is called the universal central extension. With a suitable normalization it can be given as (using the notation $L_{n}:=\left(0, l_{n}\right), t:=(1,0)$ )

$$
\left[L_{n}, L_{m}\right]=(m-n) L_{n+m}+\frac{1}{12}\left(n^{3}-n\right) \delta_{-n}^{m} t, \quad\left[t, L_{n}\right]=0, \quad \text { for all } n, m \in \mathbb{Z}
$$

(As usual, $\delta_{n}^{m}$ is the Kronecker delta.) The Lie algebra $\mathcal{V}$ defined in this way is called the Virasoro algebra.

\subsection{The Grading}

Let me point out an important fact: The Virasoro algebra becomes a graded algebra by defining

$$
\operatorname{deg}\left(L_{n}\right):=n, \quad \operatorname{deg}(t):=0
$$

Using the grading $\mathcal{V}$ decomposes into subalgebras

$$
\begin{gathered}
\mathcal{V}=\mathcal{V}_{+} \oplus \mathcal{V}_{(0)} \oplus \mathcal{V}_{-} \\
\mathcal{V}_{+}:=\left\langle L_{n} \mid n \in \mathbb{N}\right\rangle, \quad \mathcal{V}_{(0)}:=\left\langle L_{0}, t\right\rangle, \quad \mathcal{V}_{-}:=\left\langle L_{-n} \mid n \in \mathbb{N}\right\rangle .
\end{gathered}
$$

In physics one is mainly interested in highest weight representations of the Virasoro algebra, i.e. in representations which are generated from a vacuum vector $|0\rangle$ by 
$\mathcal{V}_{-}$, the vacuum is annihilated by $\mathcal{V}_{+}$, we have $L_{0}|0\rangle=h|0\rangle$ with $h \in \mathbb{C}$, and $t \psi=$ $c \cdot \psi$ for every vector $\psi$ with a fixed $c \in \mathbb{C}$. The number $c$ is called the central charge, the number $h$ the weight of the representation. The operator $\left(-L_{0}\right)$ should be considered as energy operator. Such kind of representations are "positive energy" representations, because $\left(-L_{0}\right)$ is diagonalisable and its eigenvalues are bounded from below. Note that one calculates for $\psi=L_{-n}|0\rangle$ (for $n>0$ )

$$
\left(-L_{0}\right) \psi=-L_{0} L_{-n}|0\rangle=\left(L_{-n}\left(-L_{0}\right)+\left[-L_{0}, L_{-n}\right]\right)|0\rangle=(n-h) \psi .
$$

\subsection{The Classical Sugawara Construction for the Heisenberg Algebra}

Let $H$ be the infinite-dimensional Heisenberg algebra. It is defined as the Lie algebra with underlying vector space

$$
H=\mathbb{C} \oplus\left\langle a_{n} \mid n \in \mathbb{Z}\right\rangle_{\mathbb{C}}
$$

and Lie structure

$$
\left[a_{n}, a_{m}\right]=n \delta_{-n}^{m}, \quad n, m \in \mathbb{Z}, \quad[\mathbb{C}, H]=0
$$

It is a central extension of the one-dimensional abelian Lie algebra. The bosonic Fock space is defined as

$$
F=\mathbb{C}\left[x_{1}, x_{2}, \ldots, x_{n}, \ldots\right]
$$

the space of polynomials in infinitely many variables. We define an action of $H$ on $F$ by setting

$$
\begin{gathered}
a_{n}=\frac{\partial}{\partial x_{n}}, \quad a_{-n}=n x_{n} \cdot, \quad n \in \mathbb{N} \\
a_{0}=i d, \quad t=i d .
\end{gathered}
$$

The $a_{n}$ with $n<0$ are "creation operators" in the sense that they generate all vectors (the "states") from the vacuum $|0\rangle$, which is given here by the constant function 1. The $a_{n}$ with $n>0$ are "annihilation operators" in the sense that if applied to a fixed vector suitable often they map this vector to zero. For this representation the current is defined as the formal sum of operators

$$
J(z):=\sum_{n \in \mathbb{Z}} a_{n} z^{-n-1}
$$

and the energy-momentum tensor as

$$
T(z):=-\frac{1}{2}: J(z) J(z):
$$


where we ignore the colons for a moment. Applied to an element of the Fock space the current (2.20) will produce a formal sum of elements of the Fock space. By the annihilation property, the components will vanish below a certain degree in the variable $z$ (the lower bound for the degree will depend on the vector on which the current operates). Now (2.21) should be understood in the sense that one applies $J(z)$ twice on the vector and recollects the powers of $z$ again:

$$
\begin{gathered}
T(z)=-\frac{1}{2} \sum_{k \in \mathbb{Z}}\left(\sum_{l \in \mathbb{Z}}: a_{k-l} a_{l}:\right) z^{-k-2}=\sum_{k \in \mathbb{Z}} S_{k} z^{-k-2}, \\
S_{k}:=-\frac{1}{2} \sum_{l \in \mathbb{Z}}: a_{k-l} a_{l}: .
\end{gathered}
$$

Note that $S_{k}$ is not a member of the algebra $H$. It is only an operator on the Fock space. Up to now it is not even clear whether it is a well-defined operator at all. Let $v \in F$ be a fixed polynomial, then $a_{l} v=0$ for $l \gg 0$, because there will be a maximal index of the variables in the polynomial. Note that for $k-l \neq-l$ (i.e. $k \neq 0$ ) the operators commute: $a_{k-l} a_{l}=a_{l} a_{k-l}$, see (2.17). Hence, in the defining sum (2.22) for the $S_{k}$ for $k \neq 0$ we can interchange the operators for $l \ll 0$ and only finitely many terms will survive if we apply it to the fixed vector $v$. It follows that $S_{k}(k \neq 0)$ is well-defined. If we continue to ignore the colons further on, this would not be true for $S_{0}=\sum_{l}: a_{-l} a_{l}$ :, which can easily seen by applying it to the element $1 \in F$. We would obtain $\left(-\sum_{n<0} n\right) 1$. By the colons we indicate that we use normal ordering which is defined as follows

$$
: a_{n} a_{m}::= \begin{cases}a_{n} a_{m}, & m \geq n \\ a_{m} a_{n}, & m<n .\end{cases}
$$

This forces the annihilation operators to the right. The operator $S_{0}$ becomes welldefined, the other $S_{k}$ do not change. By direct calculation we obtain

$$
\left[S_{n}, S_{m}\right]=(m-n) S_{n+m}+\frac{1}{12}\left(n^{3}-n\right) \delta_{-n}^{m} i d, \quad n, m \in \mathbb{Z} .
$$

By comparing (2.11) and (2.25) we see that we get a representation of the Virasoro algebra with central charge 1 . If we would have been able to define the action without passing to normal ordering there would be no central term. So necessarily normalization, regularization, quantization, ... forces us to consider central extensions.

\subsection{The Sugawara Construction for the Affine Lie Algebra}

The construction can be extended to representations of affine Lie algebras (i.e. KacMoody algebras of affine type). Let $\mathfrak{g}$ be a finite-dimensional Lie algebra with invariant, symmetric, non-degenerate bilinear form (.....) (i.e. $([a, b] \mid c)=(a \mid[b, c]))$.

Such a bilinear form exists for example for reductive Lie algebras, i.e. Lie algebras 
which are direct sums of simple and abelian ones. Take for the simple factors the Cartan-Killing form and for the abelian factors any non-degenerate bilinear form.

The affine Lie algebra is defined as follows. Take as vector space

$$
\widehat{\mathfrak{g}}:=\left(\mathfrak{g} \otimes \mathbb{C}\left[z, z^{-1}\right]\right) \oplus \mathbb{C} \cdot t_{1},
$$

and denote for short $x(n):=x \otimes z^{n}$ then a Lie algebra structure is defined by $(n, m \in \mathbb{Z})$

$$
[x(n), x(m)]=[x, y](n+m)+(x \mid y) n \delta_{-m}^{n} \cdot t_{1}, \quad\left[t_{1}, \widehat{\mathfrak{g}}\right]=0 .
$$

The Lie algebra without the central term is called loop algebra or current algebra. Again these algebras admit gradings defined by $\operatorname{deg}(x(n)):=n$ and $\operatorname{deg}\left(t_{1}\right)=0$.

Let $V$ be an admissible representation of $\widehat{\mathfrak{g}}$. This says that every fixed vector $v \in V$ will be annihilated by $x(n)$ for every $x \in \mathfrak{g}$ if $n$ is big enough, and that the central element $t_{1}$ operates as a scalar $c$. The scalar is called the level of the representation. Let $d=\operatorname{dim} \mathfrak{g}$, and let $\left\{u_{i}, i=1, \ldots, d\right\}$ be a basis of $\mathfrak{g}$ and $\left\{u^{i}\right\}$ the corresponding dual basis with respect to (..|..). The Casimir element of $\mathfrak{g}$ can be given as

$$
C_{\mathfrak{g}}=\sum_{i=1}^{d} u_{i} u^{i}
$$

Assume it operates as scalar on the adjoint representation, and set $\kappa$ as

$$
2 \kappa=\sum_{i=1}^{d} a d_{u_{i}} \circ a d_{u^{i}} .
$$

The assumption is for example fulfilled for $\mathfrak{g}$ abelian or $\mathfrak{g}$ a simple Lie algebra. In the abelian case clearly $\kappa=0$. If take the normalized Cartan-Killing form (the long roots have the square length 2) in the simple case then $\kappa$ equals the dual Coxeter number. For $A_{n}=\operatorname{sl}(n+1, \mathbb{C})$ one obtains $\kappa=n+1$. Assume further on that the level $c$ of the representation is such that $c+\kappa \neq 0$. Now we define

$$
S_{k}:=-\frac{1}{2(c+\kappa)} \sum_{l \in \mathbb{Z}} \sum_{i=1}^{d}: u_{i}(k-l) u^{i}(l):,
$$

with normal ordering

$$
: x(n) y(m)::= \begin{cases}x(n) y(m), & m \geq n \\ y(m) x(n), & m<n .\end{cases}
$$

The $S_{k}$ are well-defined operators on $V$ and one obtains 
Theorem 2.1. The mapping

$$
L_{k} \mapsto \quad S_{k}, \quad k \in \mathbb{Z}, \quad \text { and } \quad t \mapsto i d_{V}
$$

defines a representation of the Virasoro algebra with central charge

$$
\frac{c \cdot \operatorname{dim} \mathfrak{g}}{c+\kappa}
$$

A proof of this now classical theorem can be found for example in [12]. See also this book for references to the original proofs. Note that the Heisenberg algebra is the affinization of the one-dimensional abelian Lie algebra and that the Fock space representation of the Heisenberg algebra is an admissible representation. The Sugawara construction in Section 2.4 can be considered as special case of the general construction. But it admits a more direct and simpler proof.

\section{THE GENERALIZED KRICHEVER-NOVIKOV ALGEBRAS}

\subsection{The Geometric Set-Up}

As explained in Section 2.1 the Virasoro algebra without central term can be considered as the algebra of meromorphic vector fields on $\mathbb{P}^{1}(\mathbb{C})$ which have no poles outside $\{0, \infty\}$. The algebra $\mathbb{C}\left[z, z^{-1}\right]$ of Laurent polynomials which appear in the definition of the affine algebras is the algebra of meromorphic functions which have no poles outside $\{0, \infty\}$. Extension to higher genus means that we should replace $\mathbb{P}^{1}(\mathbb{C})$ by an arbitrary compact Riemann surface. This was started by Krichever and Novikov [13] for the two-point situation in 1987 and generalized by me 1989 [16], [17] to the multi-point situation. See also R. Dick [4] and V.A. Sadov [15] for related work. The multi-point situation is very important in the global approach to the theory of conformal blocks. You might think this generalization will be an easy thing to do. Of course, writing down the definitions of the algebras is indeed easy. One first complication (and this is not the only one) is the fact that classically we have graded algebras and we really need them for the representations. It is not possible anymore to define a honest graded structure. We will replace the grading by a weaker concept, an almost grading, which still will do the job. The second task is to find an invariant geometric description of the formal algebraic objects, like the cocycles etc.

For the following let $M$ be a compact Riemann surface, $A$ a finite set of points of $M$ which is divided into two disjoint non-empty subsets $I$ and $O: A=I \cup O$, \#I= $K \geq 1, \# O=L \geq 1, N=K+L$. The elements of $I$ are the "in-points", the elements of $O$ are the "out-points". Let $\rho$ be a meromorphic differential, holomorphic outside $A$, with exact pole order 1 at the points of $A$, (given) positive residues at $I$, (given) negative residues at $O$ (of course, obeying $\sum_{P \in M} \operatorname{res}_{P}(\rho)=0$ ). By requiring $\rho$ to have purely imaginary periods it is fixed. We fix as reference point another point 
$R \in M \backslash A$ and set

$$
u(P):=\operatorname{Re} \int_{R}^{P} \rho .
$$

It is a well-defined harmonic function. The level lines

$$
C_{\tau}=\{P \in M \backslash A \mid u(P)=\tau\}, \quad \tau \in \mathbb{R}
$$

define a fibering of $M \backslash A$. Every level line separates the in-points from the out-points. For $\tau \ll 0$ the level line $C_{\tau}$ is a disjoint union of (deformed) circles around the points in $I$. For $\tau \gg 0$ it is a disjoint union of (deformed) circles around the points in $O$. This has an interpretation in string theory. The points in $I$ correspond to free incoming strings and the points in $O$ to free outgoing strings. The variable $\tau$ might be interpreted as proper time of the string on the world sheet. The interaction of the strings is given by the geometry (genus, etc.) of the world sheet.

In the classical situation we have $g=0, I=\{0\}, O=\{\infty\}$ and

$$
\rho=\frac{1}{z} d z, \quad u(z)=\operatorname{Re} \int_{1}^{z} \frac{1}{w} d w=\log |z|
$$

Here the level lines are honest circles.

\subsection{The Almost Graded Algebras}

Let $\mathcal{L}$ be the space of meromorphic vector fields on $M$ which are holomorphic outside $A$. Under the Lie bracket of vector fields $\mathcal{L}$ becomes a Lie algebra. Let $\mathcal{K}$ be the canonical bundle, i.e. the bundle whose local sections are the local holomorphic differentials. For every $\lambda \in \mathbb{Z}$ we consider the bundle $\mathcal{K}^{\lambda}:=\mathcal{K}^{\otimes \lambda}$. Its local sections are given by the holomorphic $\lambda$-form, i.e. by local holomorphic functions which transform as they were products of $\lambda$ differentials. It is understood that for $\lambda=0$ we set $\mathcal{K}^{0}=\mathcal{O}$, the trivial bundle and for $\lambda<0, \mathcal{K}^{\lambda}:=\left(\mathcal{K}^{*}\right)^{-\lambda}$ where $\mathcal{K}^{*}$ denotes the dual bundle. ${ }^{2}$ I will adopt the common practice to use the same symbol for the bundle and its (locally free, invertible) sheaf of sections.

Let $\mathcal{F}^{\lambda}$ be the vector space of global meromorphic sections of $\mathcal{K}^{\lambda}$ which are holomorphic on $M \backslash A$. Special cases are the differentials $(\lambda=1)$, the functions $(\lambda=0)$, and the vector fields $(\lambda=-1)$. We will use $\mathcal{A}=\mathcal{F}^{0}$ and the already introduced $\mathcal{L}=\mathcal{F}^{-1}$. In the classical situation $\mathcal{A}=\mathbb{C}\left[z, z^{-1}\right]$ and $\mathcal{L}=\mathcal{W}$. The Lie algebra $\mathcal{L}$ operates on $\mathcal{F}^{\lambda}$ by taking the Lie derivative. In local coordinates the Lie derivative can be described as

$$
L_{e}(g)_{\mid}=\left(e(z) \frac{d}{d z}\right) \cdot\left(g(z) d z^{\lambda}\right)=\left(e(z) \frac{d g}{d z}(z)+\lambda g(z) \frac{d e}{d z}(z)\right) d z^{\lambda} .
$$

\footnotetext{
${ }^{2}$ After fixing a square root of the canonical bundle (a so-called theta characteristic) everything can be done for $\lambda \in \frac{1}{2} \mathbb{Z}$. For simplicity we consider here only the case of integer $\lambda$.
} 
Here I used the same symbol for the section of the bundle and its local representing function. This operation makes $\mathcal{F}^{\lambda}$ to a Lie module over $\mathcal{L}$.

It is not possible to define a graded structure. But we will be satisfied by an almost grading. This says that we can decompose $\mathcal{L}$ as direct sums of subspaces $\mathcal{L}_{n}$

$$
\mathcal{L}=\bigoplus_{n \in \mathbb{Z}} \mathcal{L}_{n}, \quad \operatorname{dim} \mathcal{L}_{n}<\infty \quad \text { and } \quad\left[\mathcal{L}_{n}, \mathcal{L}_{m}\right] \subseteq \bigoplus_{h=n+m}^{n+m+R} \mathcal{L}_{h}, n, m \in \mathbb{Z},
$$

where the integer $R$ does not depend on $n$ and $m$. The elements of $\mathcal{L}_{n}$ are called homogeneous elements of degree $n . \mathcal{L}$ is called an almost graded Lie algebra. A similar definition works for the modules.

The grading I introduce is induced by the splitting of $A$ into $I$ and $O$. Roughly, it is given by the zero orders of the forms at the points in $I$. More precisely, I exhibit basis elements of $\mathcal{F}^{\lambda}$

$$
f_{n, p}^{\lambda} \in \mathcal{F}^{\lambda}, \quad n \in \mathbb{Z}, p=1, \ldots, K=\# I
$$

and define the homogeneous subspace of degree $n$ to be

$$
\mathcal{F}_{n}^{\lambda}:=\left\langle f_{n, p}^{\lambda} \mid p=1, \ldots, K\right\rangle
$$

I do not want to write down the descriptions for the elements in all cases. They can be found in [17],[16]. To get an idea let me consider the following cases. Let $K=\# I=$ $\# O$, and let $g=0$ or $(g \geq 2$ and $\lambda \neq 0,1)$. Assume that $I=\left\{P_{1}, P_{2}, \ldots, P_{K}\right\}$, $O=\left\{Q_{1}, Q_{2}, \ldots, Q_{K}\right\}$ are points which for $g \geq 2$ are in generic position. Then there exists for every $n \in \mathbb{Z}$ and every $p=1, \ldots, K$ up to multiplication with a scalar a unique element $f_{n, p}^{\lambda} \in \mathcal{F}^{\lambda}$ with zero-orders

$$
\begin{aligned}
\operatorname{ord}_{P_{i}}\left(f_{n, p}^{\lambda}\right) & =(n+1-\lambda)-\delta_{i}^{p}, \quad i=1, \ldots, K, \\
\operatorname{ord}_{Q_{i}}\left(f_{n, p}^{\lambda}\right) & =-(n+1-\lambda), \quad i=1, \ldots, K-1, \\
\operatorname{ord}_{Q_{K}}\left(f_{n, p}^{\lambda}\right) & =-(n+1-\lambda)+(2 \lambda-1)(g-1) .
\end{aligned}
$$

After choosing local coordinates at the points in $I$ the scalar can be fixed. For the other cases the orders are only different at the points in $O$.

The elements of the basis obey the important duality relation (after fixing of the scalar)

$$
\frac{1}{2 \pi \mathrm{i}} \int_{C_{\tau}} f_{n, p}^{\lambda} \cdot f_{m, r}^{1-\lambda}=\delta_{n}^{-m} \cdot \delta_{p}^{r},
$$

where $C_{\tau}$ is any non-singular level line. If we introduce the formal infinite sum

$$
\Delta_{\lambda}\left(Q, Q^{\prime}\right):=\sum_{n \in \mathbb{Z}} \sum_{p=1}^{K} f_{n, p}^{\lambda}(Q) f_{-n, p}^{1-\lambda}\left(Q^{\prime}\right)
$$


we can interpret it (using the duality) as the delta distribution of weight $\lambda$. Indeed, we obtain for $f \in \mathcal{F}^{\lambda}$

$$
f(Q)=\frac{1}{2 \pi \mathrm{i}} \int_{C_{\tau}} f\left(Q^{\prime}\right) \Delta_{\lambda}\left(Q, Q^{\prime}\right) .
$$

Note that from the infinite sum (3.10) only finitely many terms will contribute to the calculation of the integral (3.11).

Let us consider the classical situation. Here $f_{n}^{\lambda}(z)=z^{n-\lambda} d z^{\lambda}$. With respect to the (quasi)-global coordinates $z, v$

$$
\Delta_{\lambda}(z, v)=\sum_{n \in \mathbb{Z}} z^{n-\lambda} v^{-n-(1-\lambda)} d z^{\lambda} d v^{1-\lambda} .
$$

For $\lambda=0$ we obtain

$$
\Delta_{0}(z, v)=\frac{1}{v} \sum_{n \in \mathbb{Z}} z^{n} v^{-n} d v
$$

\subsection{Central Extensions and Affine Algebras of Higher Genus}

We also have to deal with central extensions. The strategy for defining them is to find geometric descriptions of the cocycles in the classical situation which allow for generalizations to higher genus Riemann surfaces. First we consider the vector field algebra. Let $e$ and $f$ be vector fields and let us identify them with their local representing functions. Define

$$
\chi_{C, R}(e, f)=\frac{1}{24 \pi \mathrm{i}} \int_{C}\left(\frac{1}{2}\left(e^{\prime \prime \prime} f-e f^{\prime \prime \prime}\right)-R \cdot\left(e^{\prime} f-e f^{\prime}\right)\right) d z
$$

Here ' means differentiation with respect to the local coordinate $z, R$ is a meromorphic projective connection which is holomorphic in $M \backslash A$ (see Section 3.4 for its definition), and $C$ is a closed cycle in $M \backslash A$. Direct calculation [17] shows that $\chi_{C, R}$ is indeed a two-cocycle. For its value only the homology class of $C$ in $\mathrm{H}_{1}(M \backslash A, \mathbb{Z})$ is of importance. The cohomology class of $(3.14)$ in $\mathrm{H}^{2}(\mathcal{L}, \mathbb{C})$, hence the equivalence class of the central extension defined by the cocycle is independent of $R$. For the special cycles $C=C_{\tau}$ (for any $\tau$ ) the value of the integral is independent of the $\tau$ chosen. In the following we will always take $C_{\tau}$ as integration cycle and drop the cycle and the connection in the notation. By calculating residues of the integrand in (3.14) we see that there exists a constant $T$, such that for homogeneous vector fields $e$ and $f$

$$
\chi(e, f) \neq 0 \quad \Longrightarrow \quad T \leq \operatorname{deg}(e)+\operatorname{deg}(f) \leq 0 .
$$

This property is called the locality of the cocycle. For this property $C=C_{\tau}$ is essential. Using the cocycle we obtain a centrally extended vector field algebra $\widehat{\mathcal{L}}=$ $\mathbb{C} \oplus \mathcal{L}$ with

$$
[\widehat{e}, \widehat{f}]:=\widehat{[e, f]}+\chi(e, f) \cdot t
$$


using the above introduced notation $\widehat{e}=(0, e)$ and $t=(1,0)$. By $\operatorname{setting} \operatorname{deg}(\widehat{e}):=$ $\operatorname{deg}(e)$ and $\operatorname{deg}(t):=0$ we can extend the grading of $\mathcal{L}$ to $\widehat{\mathcal{L}}$. The locality of the cocycle implies that $\widehat{\mathcal{L}}$ is almost graded.

Let us now consider the (associative) algebra of functions $\mathcal{A}$. It is again an almost graded algebra, i.e. it admits a decomposition $\mathcal{A}=\bigoplus_{n \in \mathbb{Z}} \mathcal{A}_{n}, \operatorname{dim} \mathcal{A}_{n}<\infty$ with a constant $S$ such that for all $n, m \in \mathbb{Z}$

$$
\mathcal{A}_{n} \cdot \mathcal{A}_{m} \subseteq \bigoplus_{h=n+m}^{n+m+S} \mathcal{A}_{h}
$$

Let $\mathfrak{g}$ be a Lie algebra and (..|..) an invariant, non-degenerate, symmetric bilinear form on $\mathfrak{g}$ as in Section 2.5 (for example, $\mathfrak{g}$ reductive). Again the space $\mathcal{G}=\mathfrak{g} \otimes \mathcal{A}$ carries a Lie structure. We will need its central extension

$$
\begin{gathered}
\widehat{\mathcal{G}}=(\mathfrak{g} \otimes \mathcal{A}) \oplus \mathbb{C} \cdot t_{1}, \\
{[x \otimes g, y \otimes h]=[x, y] \otimes g \cdot h-\left((x \mid y) \frac{1}{2 \pi \mathrm{i}} \int_{C} g d h\right) \cdot t_{1}, \quad\left[t_{1}, \widehat{\mathcal{G}}\right]=0 .}
\end{gathered}
$$

Here I identified $x \otimes g$ with $(x \otimes g, 0)$.

In the following we will only consider extensions obtained by integrating over a level line $C=C_{\tau}$. By defining

$$
\widehat{\mathcal{G}}_{n}:=\mathfrak{g} \otimes \mathcal{A}_{n}, \quad n \in \mathbb{Z}, n \neq 0, \quad \widehat{\mathcal{G}}_{0}:=\left(\mathfrak{g} \otimes \mathcal{A}_{0}\right) \oplus \mathbb{C} t_{1},
$$

we obtain again an almost graded Lie algebra.

Note that for $\mathfrak{g}=\mathbb{C}$ and $(\alpha \mid \beta)=\alpha \beta$ we obtain a central extension $\widehat{\mathcal{A}}$ of the abelian Lie algebra $\mathcal{A}$. It is a nice and easy exercise to show that in the classical situation everything reduces to the well-known setting introduced in Section 2. By the almost-grading we obtain a decomposition

$$
\widehat{\mathcal{G}}=\widehat{\mathcal{G}}_{+} \oplus \mathcal{G}_{(0)} \oplus \widehat{\mathcal{G}}_{-},
$$

with

$$
\widehat{\mathcal{G}}_{+}=\bigoplus_{n>0} \widehat{\mathcal{G}}_{n}, \quad \widehat{\mathcal{G}}_{(0)}=\bigoplus_{n=-S, \ldots, 0} \widehat{\mathcal{G}}_{n}, \quad \widehat{\mathcal{G}}_{-}=\bigoplus_{n<-S} \widehat{\mathcal{G}}_{n}
$$

The constant $S$ is the constant appearing in (3.17). The subspaces $\widehat{\mathcal{G}}_{+}$and $\widehat{\mathcal{G}}_{-}$are subalgebras of $\widehat{\mathcal{G}}$ and can be identified with subalgebras of $\mathcal{G}$. For the two-point case the affine algebras have been introduced in [13] and its representation theory has been studied in [22]. The multi-point generalization was introduced in [18]. 


\subsection{Definition of a Projective Connection}

Let $\left(U_{\alpha}, z_{\alpha}\right)_{\alpha \in J}$ be a covering of the Riemann surface by holomorphic coordinates, with transition functions $z_{\beta}=f_{\beta \alpha}\left(z_{\alpha}\right)=h\left(z_{\alpha}\right)$. A system of local (holomorphic, meromorphic) functions $R=\left(R_{\alpha}\left(z_{\alpha}\right)\right)$ is called a (holomorphic, meromorphic) projective connection if it transforms as

$$
R_{\beta}\left(z_{\beta}\right) \cdot\left(h^{\prime}\right)^{2}=R\left(z_{\alpha}\right)+S(h), \quad \text { with } \quad S(h)=\frac{h^{\prime \prime \prime}}{h^{\prime}}-\frac{3}{2}\left(\frac{h^{\prime \prime}}{h^{\prime}}\right)^{2},
$$

the Schwartzian derivative. Here ' denotes differentiation with respect to the coordinate $z_{\alpha}$. Note that the difference of two projective connections is always a usual quadratic differential.

The following result can be found in [8]: There exists always a holomorphic projective connection.

\section{THE SUGAWARA CONSTRUCTION FOR HIGHER GENUS}

\subsection{The Results}

The following results have been obtained in joint work with Oleg K. Sheinman [20]. Let $V$ be an admissible module of the affine algebra $\widehat{\mathcal{G}}$ for higher genus. This says that the central element operates as scalar, the level of the representation, and that

$$
\text { for all } v \in V: \quad \widehat{\mathcal{G}}_{n} v=0 \text { for } n \gg 0 \text {. }
$$

Examples of such representations are the highest weight representations studied by Sheinman [22].

Again we have to choose a normal ordering :...: similar to (2.31). For $x \in \mathfrak{g}$ we define the associated current $\widetilde{x}(Q)$ to be

$$
\widetilde{x}(Q)=\sum_{n \in \mathbb{Z}} \sum_{p=1}^{K} x(n, p) \omega^{n, p}(Q)
$$

Here I used the notation

$$
e_{n, p}:=f_{n, p}^{-1}, \quad a_{n, p}:=f_{n, p}^{0}, \quad \omega^{n, p}:=f_{-n, p}^{1}, \quad \Omega^{n, p}:=f_{-n, p}^{2},
$$

(note the inverted index for $\omega^{n, p}$ and $\Omega^{n, p}$ ) and $x(n, p)$ for the operator corresponding to $x \otimes a_{n, p}$. In the following, sums over the indices of the basis elements will occur. To simplify the formulas I will drop the range of the summation if it is clear from the situation. In particular, the summation for the first index is always over $\mathbb{Z}$, for the second from 1 to $K$. Concerning the Lie algebra $\mathfrak{g}$ recall the notations introduced in Section 2.5. 
The Sugawara field or energy-momentum field is defined as

$$
T(Q):=\frac{1}{2} \sum_{i=1}^{\operatorname{dim} \mathfrak{g}}: \widetilde{u}_{i}(Q) \widetilde{u^{i}}(Q):
$$

If we plug in the definition of the currents we obtain

$$
T(Q)=\frac{1}{2} \sum_{n, m, p, r} \sum_{i}: u_{i}(n, p) u^{i}(m, r): \omega^{n, p}(Q) \omega^{m, r}(Q) .
$$

If $T(Q)$ is well-defined at all it has to be a form of weight 2 (a quadratic differential) in $Q$. Hence we can write it as the sum

$$
T(Q)=\sum_{k, s} L_{k, s} \Omega^{k, s}(Q)
$$

with certain operators $L_{k, s}, k \in \mathbb{Z}, s=1, \ldots, K$ on the representation space. To calculate the operators we use duality (3.9)

$$
L_{k, s}=\frac{1}{2 \pi \mathrm{i}} \int_{C_{\tau}} T(Q) e_{k, s}(Q)=\sum_{n, m, p, r} \sum_{i}: u_{i}(n, p) u^{i}(m, r): l_{(k, s)}^{(n, p),(m, r)}
$$

with

$$
l_{(k, s)}^{(n, p),(m, r)}=\frac{1}{2 \pi \mathrm{i}} \int_{C_{\tau}} \omega^{n, p} \omega^{m, r} e_{k, s}
$$

By considering the poles of the integrand we see for fixed $k$ that $l_{(k, s)}^{(n, p),(m, r)} \neq 0$ is possible only for a finite range of values of the sum $m+n$. E.g. for $\# I=\# O=1$ one obtains as range: $k \leq m+n \leq k+g$. Altogether we still obtain infinitely many non-vanishing coefficients. But by the normal ordering the operators with index $m \gg 0$ or $n \gg 0$ (corresponding to $m \ll 0$ ) will be moved to the right and a fixed vector $v$ will be annihilated by them. Applied to a fixed $v$ only finitely many terms will survive in the result. Hence,

Proposition 4.1. The operators $L_{k, s}$ are well-defined linear operators on an admissible representation space $V$.

Note that these operators are not elements of the affine algebra.

The key result is the following

Theorem 4.1. [20, Prop. 3.2, Prop. A.1] Let $c$ be the level of the representation and let $\kappa$ be defined by (2.29) then

$$
\left[L_{k, s}, x(n, p)\right]=-(c+\kappa) x\left(\nabla_{e_{k, s}} a_{n, p}\right) .
$$


By $x\left(\nabla_{e_{k, s}} a_{n, p}\right)$ we understand the following: take the Lie derivative of the function $a_{n, p}$ with respect to the vector field $e_{k, s}$, tensorize the resulting function with the element $x \in \mathfrak{g}$ and take the corresponding operator on $V$.

Theorem 4.2. [20, Thm. 3.1, Thm. A.1] Let $\mathfrak{g}$ be a finite-dimensional Lie algebra such that the Casimir element of $\mathfrak{g}$ acts as $2 \kappa \cdot i d$ on the adjoint representation (which is for example the case for $\mathfrak{g}$ abelian or simple). Let $V$ be an admissible representation of level c of the affine algebra $\widehat{\mathcal{G}}$. Assume $c+\kappa \neq 0$. Set

$$
L_{k, s}^{*}:=\frac{-1}{c+\kappa} L_{k, s}
$$

then the map

$$
\widehat{e}_{k, s} \mapsto \quad L_{k, s}^{*}, \quad k \in \mathbb{Z}, s=1, \ldots, K
$$

defines a representation of a central extension of the (Krichever-Novikov) vector field algebra $\widehat{\mathcal{L}}$ given by the above introduced local geometric cocycle (3.14) (with a suitable projective connection) with central charge

$$
c_{\widehat{\mathcal{L}}}=\frac{c \cdot \operatorname{dim} \mathfrak{g}}{c+\kappa} .
$$

( $\widehat{e}_{k, s}$ is the lift of the vector field $e_{k, s}$ ).

For the proof see [20], [19], resp. the comments below. The construction does depend on the normal ordering in the sense that a different choice as (2.31) would change the defining cocycle for the central extension. But in [20] it is shown that a different normal ordering does not change the cohomology class of the cocycle. Hence, we obtain equivalent central extensions.

Theorem 4.2 specializes to Theorem 2.1 in the classical situation. In addition, it gives a geometric proof for it. For $g \geq 1$ and two points the abelian (Heisenberg algebra) case was considered by Krichever and Novikov [13]. For the case that $\mathfrak{g}$ is simple and two points the situation was studied by the physicists Bonora, Rinaldi, Rosso and $\mathrm{Wu}[2]$. There the presentation was not so that every mathematician might accept it. So we reproved it for this situation, insuring that their result was correct, obtained finer results and generalized it to the multi-point situation. We felt us inspired by [2].

\subsection{Few Comments on the Proofs}

Here I will only point out some ideas of the proofs. For notational reason I consider the two point case and drop everywhere the second index. Choose a cut-off function $\psi$ such that $\psi(x)=1$ for $|x| \leq 1$ and $\psi(x)=0$ for $|x|>1, x \in \mathbb{R}$. We set

$$
L_{k}(\epsilon)=\sum_{n, m} \sum_{i}: u_{i}(n) u^{i}(m): l_{k}^{n, m} \psi(\epsilon n) \text {. }
$$


If we apply $L_{k}$ to a fixed $v \in V$ only finitely many terms in the sum will contribute. Hence, we get $L_{k} v=L_{k}(\epsilon) v$ for $|\epsilon|$ small enough. The advantage is that as long as $\epsilon \neq 0$ we can for the evaluation of the commutator

$$
\left[L_{k}(\epsilon), x(r)\right]
$$

forget about the normal ordering. Now

$$
\left[u_{i}(n) u^{i}(m), x(r)\right]=u_{i}(n)\left[u^{i}(m), x(r)\right]+\left[u_{i}(n), x(r)\right] u^{i}(m)
$$

resolves the commutators in (4.14). This gives terms connected with the level $c$ coming from the action of the central element and terms of the type

$$
\sum_{n, m, s} \sum_{i} u_{i}(n)\left[u^{i}, x\right](s) \alpha_{m, r}^{s} l_{k}^{n, m} \psi(\epsilon n)
$$

where the $\alpha_{m, r}^{s}$ are defined by $a_{m} \cdot a_{r}=\sum_{s} \alpha_{m, r}^{s} a_{s}$. For $\epsilon \rightarrow 0$ the split infinite sums considered separately do not make sense. Before we can let $\epsilon \rightarrow 0$ we have to rearrange things and pass over to normal ordering again. This gives additional terms of the type

$$
\sum_{i}\left[u_{i},\left[u^{i}, x\right]\right]
$$

And this introduces $\kappa$ as half of the eigenvalue of the Casimir operator in the adjoint representation. We also make essential use of the duality and the properties of the delta distribution $\Delta_{\lambda}$.

To prove Theorem 4.2 we proceed in a similar manner. Again consider $\left[L_{n}(\epsilon), L_{m}\right]$. By the cut-off we can forget about the normal ordering, do the calculation (using Theorem 4.1, duality, etc.) in a non-straight-forward manner. Changing back to normal ordering to make things well-defined for $\epsilon \rightarrow 0$ will give contributions to the central term.

The details can be found in [20] and [19].

\subsection{Further Results}

(a) For the higher genus algebras by the almost-grading it is possible to introduce a notion of highest weight representations. In particular, the highest weight representation is an admissible representation. In the classical situation the weight for an affine algebra is an element of the dual of the Cartan subalgebra. In the higher genus the weight has more components. For the two-point situation this was developed by O.K. Sheinman [22]. There you can also find the classification of the representations. For the multi-point case see [19]. If we start with highest weight representations of the affine algebra $\widehat{\mathcal{G}}$ we obtain highest weight representations for the vector field algebra. In the article [20] relations between the involved weights are given. 
(b) For admissible representations Theorem 4.1 allows to consider the semi-direct product of the operator algebra corresponding to $\widehat{\mathcal{G}}$ and the algebra obtained by the Sugawara operators $L_{k, s}^{*}$ (4.10) using the rescaled action (4.9) of $L_{k, s}^{*}$ on $x(n, p)$. The representation of the affine algebra will naturally extend to a representation of the semi-direct product.

(c) Under additional assumptions on the representations it is possible to construct Casimir operators for representations of the affine algebra [20].

\section{References}

[1] A.A. Belavin, A.M. Polyakov, A.B. Zamolodchikov, Infinite conformal symmetry in twodimensional quantum field theory, Nucl. Phys. B241, 333-380 (1984)

[2] L. Bonora, M. Rinaldi, J. Russo, K. Wu, The Sugawara construction on genus $g$ Riemann surfaces, Phys. Lett. B 208, 440-446 (1988)

[3] J-L.Brylinski,D. McLaughlin, Holomorphic quantization and unitary representations of the Teichmüller group in: "Lie theory and geometry, in honor of B. Kostant", eds. J-L. Brylinski, R. Brylinski, V. Guillemin, V. Kac, p. 21-64, Birkhäuser, Boston (1994)

[4] R. Dick, Krichever-Novikov-like bases on punctured Riemann surfaces, Lett. Math. Phys. 18, $255-265$ (1989)

[5] T. Eguchi, H. Ooguri, Conformal and current algebras on a general Riemann surface, Nucl. Phys. B282, 308-328 (1987)

[6] D. Friedan, S. Shenker, The analytic geometry of two-dimensional conformal field theory, Nucl. Phys. B281, 509-545 (1987)

[7] I.M. Gelfand, D.B. Fuks, Cohomology of the Lie algebra of vector fields on a circle, Funktional Anal. Appl. 2, 342-343 (1968);

[8] R.C. Gunning, "Lectures on Riemann surfaces", Princeton Mathematical Notes, Princeton University Press, Princeton, N.J., USA (1966)

[9] M.B. Halpern, E. Kiritsis, N. Obers, K. Clubok, Irrational Conformal Field Theory, hepth/9501144, Phys. Rept. 265, 1-138 (1996)

[10] P.J. Hilton, U. Stammbach, "A Course in Homological Algebra", Springer, New YorkHeidelberg-Berlin (1971)

[11] V. Kac, "Infinite dimensional Lie algebras", Cambridge University Press, Cambridge (1990)

[12] V. Kac, A.K. Raina, "Highest Weight Representations of Infinite Dimensional Lie Algebras", World Scientific, Singapore, London (1987)

[13] I.M. Krichever, S.P. Novikov, Algebras of Virasoro type, Riemann surfaces and structures of the theory of solitons, Funktional Anal. Appl. 21(2), 46 (1987); Virasoro type algebras, Riemann surfaces and strings in Minkowski space, Funktional Anal. Appl. 21(4), 47 (1987); Algebras of Virasoro type, energy-momentum tensors and decompositions of operators on Riemann surfaces, Funktional Anal. Appl. 23(1), 19 (1989) 
[14] A. Pressley, G. Segal "Loop Groups", Oxford Science Publications, Oxford (1986)

[15] V.A. Sadov, Bases on multipunctured Riemann surfaces and interacting strings amplitudes, Commun. Math. Phys. 136, 585-597 (1991)

[16] M. Schlichenmaier, Krichever-Novikov algebras for more than two points, Lett. Math. Phys. 19, 151-165 (1990); Krichever-Novikov algebras for more than two points: explicit generators, Lett. Math. Phys. 19, 327-336 (1990); Central extensions and semi-infinite wedge representations of Krichever-Novikov algebras for more than two points, Lett. Math. Phys. 20, 33-46. (1990)

[17] M. Schlichenmaier, "Verallgemeinerte Krichever-Novikov Algebren und deren Darstellungen", Dissertation, Universität Mannheim (1990)

[18] M. Schlichenmaier, Differential operator algebras on compact Riemann surfaces, in: "Generalized Symmetries in Physics", H.-D. Doebner, V.K. Dobrev, A.G Ushveridze eds., World Scientific, Singapore, London (1994)

[19] M. Schlichenmaier, "Zwei Anwendungen algebraisch-geometrischer Methoden in der theoretischen Physik: Berezin-Toeplitz-Quantisierung und globale Algebren der zweidimensionalen konformen Feldtheorie", Universität Mannheim (1996)

[20] M. Schlichenmaier, O.K. Sheinman, Sugawara construction and Casimir operators for Krichever-Novikov algebras, Mannheimer Manuskripte Nr. 201 und q-alg/9512016 (1995) (to appear in Jour. of Math. Sciences, Vol. 38 (Itogi Nauki))

[21] M. Schlichenmaier, O.K. Sheinman, Wess-Zumino-Witten-Novikov theory, KnizhnikZamolodchikov equations and Krichever-Novikov algebras, (in preparation)

[22] O.K. Sheinman, Elliptic affine Lie algebras, Funktional Anal. Appl. 24(3), 210-219 (1990); Highest weight modules over certain quasigraded Lie algebras on elliptic curves, Funktional Anal. Appl. 26(3), 203-208 (1992); Affine Lie algebras on Riemann surfaces, Funktional Anal. Appl. 27(4), 54-62 (1993); Highest weight modules for affine Lie algebras on Riemann surfaces, Funktional Anal. Appl. 29(1), 56-71 (1995);

[23] H. Sugawara, A field theory of currents, Phys. Rev., 176, 2019-2025 (1968)

[24] A. Tsuchiya, K. Ueno, Y. Yamada, Conformal field theory on universal family of stable curves with gauge symmetries, Adv. Stud. Pure Math. 19, 459-566 (1989) 